Article

\title{
In-Situ Arc Discharge-Derived FeSn 2 /Onion-Like Carbon Nanocapsules as Improved Stannide-Based Electrocatalytic Anode Materials for Lithium-Ion Batteries
}

\author{
Dandan Han ${ }^{1,2,3}$, Amrita Chatterjee ${ }^{1,2}$, Long Hin Man ${ }^{1,2}$ and Siu Wing Or 1,2,*(D) \\ 1 Department of Electrical Engineering, The Hong Kong Polytechnic University, Hung Hom, Kowloon, \\ Hong Kong, China; loveh_andan@163.com (D.H.); achatterjee@connect.ust.hk (A.C.); \\ kelvin123456.man@connect.polyu.hk (L.H.M.) \\ 2 Hong Kong Branch of National Rail Transit Electrification and Automation Engineering Technology \\ Research Center, Hong Kong, China \\ 3 College of Science, Northeast Electric Power University, Jilin 132012, China \\ * Correspondence: eeswor@polyu.edu.hk; Tel.: +852-3400-3345; Fax: +852-2330-1544
}

Received: 21 October 2019; Accepted: 6 November 2019; Published: 13 November 2019

\begin{abstract}
Core/shell-structured FeSn 2 /onion-like carbon ( $\left.\mathrm{FeSn}_{2} / \mathrm{OLC}\right)$ nanocapsules of confined size range of sub-50 $\mathrm{nm}$ are synthesized via an in-situ arc-discharge process, and are evaluated in comparison with $\mathrm{FeSn}_{2}$ nanoparticles as an improved stannide-based electrocatalytic anode material for Li-ion batteries (LIBs). The in-situ arc-discharge process allows a facile one-pot procedure for forming crystalline $\mathrm{FeSn}_{2}$ stannide alloy nanoparticle cores coated by defective OLC thin shells in addition to a confined crystal growth of the FeSn 2 nanoparticle cores. The LIB cells assembled using the $\mathrm{FeSn}_{2} / \mathrm{OLC}$ nanocapsules as the electrocatalytic anodes exhibit superior full specific discharge capacity of $519 \mathrm{mAh} \cdot \mathrm{g}^{-1}$ and specific discharge capacity retention of $\sim 62.1 \%$ after 100 charge-discharge cycles at $50 \mathrm{~mA} \cdot \mathrm{g}^{-1}$ specific current. The electrochemical stability of $\mathrm{FeSn}_{2} / \mathrm{OLC}$ nanocapsules is demonstrated from the good cycle stability of the LIBs with a high specific discharge capacity retention of $67.5 \%$ on a drastic change in specific current from 4000 to $50 \mathrm{~mA} \cdot \mathrm{g}^{-1}$. A formation mechanism is proposed to describe the confined crystal growth of the FeSn 2 nanoparticle cores and the formation of the FeSn $2 / \mathrm{OLC}$ core/shell structure. The observed electrochemical performance enhancement is ascribed to the synergetic effects of the enabling of a reversible lithiation process during charge-discharge of the LIB cells by the FeSn 2 nanoparticle cores as well as the protection of the $\mathrm{FeSn}_{2}$ nanoparticle cores from volume change-induced pulverization and solid electrolyte interphase-induced passivation by the OLC shells.
\end{abstract}

Keywords: core/shell-structured nanocapsules; FeSn 2 stannide alloys; in-situ arc-discharge process; onion-like carbon; electrocatalytic; Li-ion batteries

\section{Introduction}

Lithium (Li)-ion batteries (LIBs) have become the most popular class of energy storages and power sources for portable electronics and mobile devices because of their high energy density, rate capability, cycle stability, and market availability [1,2]. To cope with the rapidly increasing challenges by other classes of energy storages and power sources, it is urgent to develop higher performance electrocatalytic anodes to extend the application scopes of LIBs, especially in the fast-growing fields of electric vehicles and renewable energies [3,4]. Carbonaceous materials such as graphite have been investigated as anodes for LIBs without much success because the $\mathrm{Li}^{+}$ions stored in the 2-D 
carbon planes can form a maximum stoichiometry of $\mathrm{LiC}_{6}$, thus yielding a low theoretical specific discharge capacity $\left(372 \mathrm{mAh} \cdot \mathrm{g}^{-1}\right)$, low discharge voltage $(<0.2 \mathrm{~V})$, sluggish reaction kinetics, and ease of formation of dendritic side products $[1,5]$. In contrast, tin (Sn) has shown the ability to form reversible and electrochemical alloying reaction with $\mathrm{Li}$ up to $\mathrm{Li}_{4.4} \mathrm{Sn}$, thus providing higher lithium storage capacity and consequently higher theoretical specific discharge capacity of $993 \mathrm{mAh} \cdot \mathrm{g}^{-1}$ in comparison with $372 \mathrm{mAh} \cdot \mathrm{g}^{-1}$ for the conventional graphite [6,7]. Sn also gains merits as the choicest candidate for electrocatalytic anodes because of their high availability, low cost, and high electrical conductivity. In 2005, Sony's Nexelion battery composed of amorphous Sn-Co-C as electrocatalytic anode gave the first breakthrough of the positive electrocatalytic influences of Sn-based or "stannide" intermetallic alloys for LIBs, and opened up a globally significant research direction in exploring stannide intermetallic alloys as promising commercial electrocatalytic anodes for LIBs $[5,7]$.

Among the various stannide intermetallic alloys that have been reported, $\mathrm{SnCo}, \mathrm{CoSn}_{2}$, and $\mathrm{FeSn}_{2}$ have shown favorable electrocatalytic properties as LIB anodes [8,9]. However, the cyclability and specific discharge capacity of these stannide alloys, more specifically $\mathrm{FeSn}_{2}$ is still far from the state-of-the-art. In this context, three major drawbacks have been identified. First, the large volume change of $\mathrm{FeSn}_{2}$ alloy nanoparticle during the lithiation-delithiation process induce increasing inner stress, that cause pulverization of the active electrocatalyst, thereby inducing loss of electrical connection with the current collectors $[2,10,11]$. Though stannide alloy anodes with Fe or Co as electrocatalytically inactive act as buffering agent (e.g., $\mathrm{FeSn}_{2}$ and $\mathrm{CoSn}_{2}$ ) are capable of improving the cyclability of the LIBs by redistributing the volume change-induced fragmentation, yet the minimization of pulverization of the nanoparticles is still a major concern that needs to be addressed [2,12]. Second, the new surfaces formed by the pulverized active electrocatalyst consume higher amount of $\mathrm{Li}$, leading to the formation of solid-electrolyte interphase (SEI) that subsequently passivates the anode and increases the undesired electrochemical resistance [11]. Finally, the electrical conductivity of the stannide-alloys is not comparable to the state-of-the-art LIB anode materials $[2,10,12,13]$.

To overcome the above-mentioned drawbacks of $\mathrm{FeSn}_{2}$ alloy nanoparticles and design them as improved electrocatalytic LIB anodes, it is necessary to synthesize precisely controlled sub-50 $\mathrm{nm}$-sized crystalline $\mathrm{FeSn}_{2}$. Previous results suggested that lack of intrinsic and extrinsic defects in highly crystalline $\mathrm{FeSn}_{2}$ alloy nanoparticles prevent the irreversible trapping of $\mathrm{Li}^{+}$ions, resulting in better cyclability and lower initial discharge capacity compared to their bulk counterparts [12,14]. The sub-50 nm size crystalline $\mathrm{FeSn}_{2}$ would also possibly alleviate the absolute strain at nanoscale and reduce the charge-diffusion pathways. But the lack of such precisely controlled sub-50 nm-sized crystalline $\mathrm{FeSn}_{2}$ alloy nanoparticles have impeded their performance as LIB anodes. The conductivity of these stannide alloy anodes can be improved by compositing them with carbon, which have also been proven beneficial to accommodate strains during the lithiation-induced volume change and minimize pulverization of the core $\mathrm{FeSn}_{2}$ nanoparticles [2,13]. However, the current syntheses procedures are inadequate in introducing all these three features efficiently, that result in the poor electrochemical performance of stannide-based intermetallic alloys, more specifically $\mathrm{FeSn}_{2}$, as electrocatalytic LIB anodes.

Among the various syntheses procedures adopted to incorporate the above-mentioned features in electrocatalytic stannide-based intermetallic alloys are: mechanically milled $\mathrm{FeSn}_{2} / \mathrm{C}$ nanocomposites that exhibited specific discharge capacity of $400 \mathrm{mAh} \cdot \mathrm{g}^{-1}$ and stability till 50 charge-discharge cycles [9]; $\mathrm{Sn}-\mathrm{Fe} / \mathrm{C}$ nanocomposites synthesized by mechanochemical method showed a low specific discharge capacity of $380 \mathrm{mAh} \cdot \mathrm{g}^{-1}$ till 100 cycles; $\mathrm{FeSn}_{2}$ anodes prepared by thermal treatment of precursors provided $500 \mathrm{mAh} \cdot \mathrm{g}^{-1}$ specific discharge capacity and cycle stability till 50-60 cycles [15]; $\mathrm{FeSn}_{2}$ prepared by surfactant assisted solvothermal method could discharge till $1268 \mathrm{mAh} \cdot \mathrm{g}^{-1}$, but their cyclability rapidly dropped by the 20th cycle [6]; multiphase Fe-Sn/C synthesized by arc-discharge process using methanol as precursor, showed specific discharge capacity of $818 \mathrm{mAh} \cdot \mathrm{g}^{-1}$, only till 50 cycles [2]. Though $\mathrm{FeSn}_{2}$ nanocrystals synthesized by a successive reduction process delivered a specific discharge capacity of $\sim 600 \mathrm{mAh} \cdot \mathrm{g}^{-1}$ for 500 cycles, but the excess use of hazardous 
toluene and chloroform and expensive reagents like lithium di-isopropylamide [16] undermines their commercialization and environment aspects. Apart from the multiple steps used in the above methods, the moderate electrochemical performance of these $\mathrm{FeSn}_{2}$ alloys might be attributed to the controlled morphology of the active sites, their electrochemical instability during for lithiation-delithiation process, and the failure of the fabricated carbon shell to accommodate the discharge products.

Further, the previous works on electrocatalytic $\mathrm{FeSn}_{2} / \mathrm{carbon}$ composite uses the conventional carbon matrix. But recent works on onion-like carbon shell (OLC) in supercapacitors and microwave absorbing devices prove their superiority over conventional carbon matrix $[17,18]$. OLC are nanoparticles with quasi-spherical shape composed of multiple enclosed fullerene-like defective carbon shells with diameters between 5-10 nm [19]. Owing to their surface defective features, they exhibit superior electrical conductivity, structural flexibility, and full accessibility of ion adsorption/desorption on their surface [20].

To address the lack of controlled and environmentally benign synthesis process for strategically designed electrocatalytic stannide-based intermetallic alloys for LIB anodes, in this work we have used a facile one-pot in-situ arc-discharge process to synthesize pure-phase $\mathrm{FeSn}_{2} / \mathrm{OLC}$ nanocapsules, featuring a confined crystal size range of sub-50 $\mathrm{nm}$ and attractive electrochemical properties of core/shell-structured Sn-based stannide alloys. The high controllability achieved by arc-discharge method enables the introduction of Fe as the buffering metal, development of sub-50 $\mathrm{nm}$ size intermetallic $\mathrm{FeSn}_{2}$ alloys, and their encapsulation by OLC shells with surface defective features. Moreover, the tactful combination of crystalline $\mathrm{FeSn}_{2}$ nanoparticles and OLC improve the electrochemical stability of the nanocapsules and enable a reversible lithiation process during charge-discharge of the LIB cells with high specific discharge capacity $835 \mathrm{mAh} \cdot \mathrm{g}^{-1}$ and stability till 100 cycles. To the best of our knowledge there is no report on the synthesis of pure-phase electrocatalytic $\mathrm{FeSn}_{2} / \mathrm{OLC}$ nanocapsules by a scalable, in-situ arc-discharge method to serve as the LIB anodes.

\section{Results and Discussion}

\subsection{Physicochemical Properties of FeSn $/ 2$ OLC Nanocapsules}

The XRD pattern of the $\mathrm{FeSn}_{2} / \mathrm{OLC}$ nanocapsules in Figure 1a reveals a tetragonal unit cell with C16 type structure (JCPDS no. 25-0415, space group I4/mcm, $a=b=6.538 \AA, c=5.326 \AA$ ) of the core $\mathrm{FeSn}_{2}$ nanoparticles which corroborate with stannide structure [21,22]. The diffraction peaks at $27.3^{\circ}$, $33.7^{\circ}, 35.1^{\circ}, 39.1^{\circ}, 43.8^{\circ}, 56.4^{\circ}, 61.1^{\circ}, 63.7^{\circ}, 67.2^{\circ}$, and $70.8^{\circ}$ correspond to (200), (002), (211), (112), (202), (312), (213), (420), (402), and (004) Millers indices [23]. According to Armbuster et al. the FeSn 2 stannide structure is formed of two types of building blocks. Because of the homo-atomic interactions of the tin atoms along $d 1$ and $d 2$, a $6^{3}$ interpenetrating net-like structure is formed. On the other hand, because of the covalent interactions, the Fe atoms constitute chains along (001). Both of these building blocks are connected by the $\mathrm{Fe}-\mathrm{Sn}-\mathrm{Fe}$ heteroatomic bonds and eventually gives rise to a three dimensional network as seen in Figure 1b [16]. The absence of any other peaks indicates the high purity of the $\mathrm{FeSn}_{2} / \mathrm{OLC}$ nanocapsules devoid of any oxidized metal products when prepared by arc-discharge method. Because of the small amount of carbon present in the FeSn 2 /OLC nanocapsules, no evident peak of carbon is observed. There is a possibility of breaking down of the periodic boundary condition of the carbon shells (translation symmetry) along the radial direction which make them undetectable in the XRD patterns [24]. The slight left shift of the diffraction peaks of $\mathrm{FeSn}_{2} / \mathrm{OLC}$ nanocapsules as compared to pure $\mathrm{FeSn}_{2}$ nanoparticles is attributed to the doping of the atoms. Since the preferred orientation of $\mathrm{FeSn}_{2}$ is in the (211) direction [16,22,23], therefore the crystal size is calculated from the Scherrer's equation considering this peak. It is estimated that the average crystal size of the $\mathrm{FeSn}_{2}$ nanoparticles was $27.3 \mathrm{~nm}$. On the other hand, the $\mathrm{FeSn}_{2}$ core of the $\mathrm{FeSn}_{2} / \mathrm{OLC}$ nanocapsules possesses an average crystal size of $23.6 \mathrm{~nm}$. The reduction in crystal size is evident from the broadness of the (211) peak of $\mathrm{FeSn}_{2} / \mathrm{OLC}$ nanocapsules. Hence, it is concluded that the OLC shells suppress the growth of the $\mathrm{FeSn}_{2}$ nanoparticles to sub-50 nm, thereby improving their electrochemical properties. 

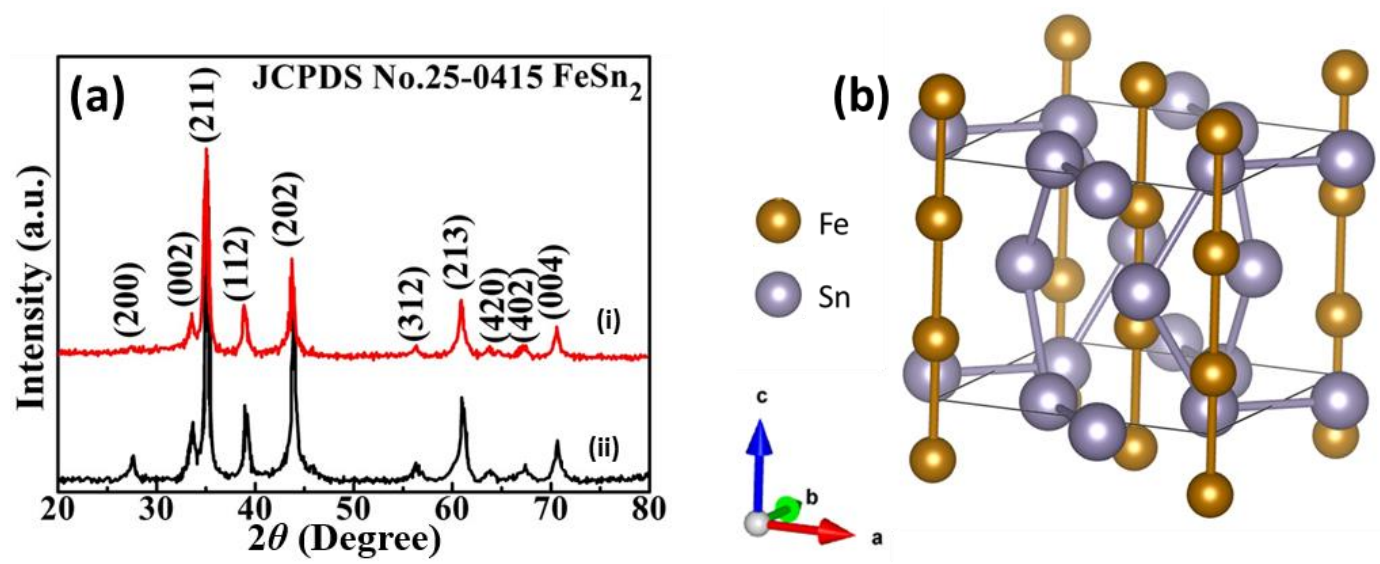

Figure 1. (a) XRD patterns of (i) FeSn 2 /onion-like carbon ( $\mathrm{FeSn}_{2} / \mathrm{OLC}$ ) nanocapsules and (ii) $\mathrm{FeSn}_{2}$ nanoparticles. (b) Crystal structure of $\mathrm{FeSn}_{2}$.

The HR-TEM images in Figure 2a show the spherically shaped FeSn 2 nanoparticles with diameter ranging from $5-120 \mathrm{~nm}$. The formation of $\mathrm{FeSn}_{2}$ phase is confirmed from the characteristic lattice fringe of $d=0.256 \mathrm{~nm}$ as in Figure 2c, corresponding to the lattice plane of (211). Figure 2b exhibits the core/shell structure of $\mathrm{FeSn}_{2} / \mathrm{OLC}$ nanocapsules after the in-situ arc-discharge process. The $\mathrm{FeSn}_{2} / \mathrm{OLC}$ nanocapsules retain their spherical shapes with distinct onion like thin shells of $\sim 1 \mathrm{~nm}$ thickness on the outer perimeter. Because of the suppression of the crystal growth in $\mathrm{FeSn}_{2} / \mathrm{OLC}$ nanocapsules, a narrow size distribution of the nanocapsules is achieved within $5-50 \mathrm{~nm}$ range. The onion like shells exhibits a lattice fringe of $d=0.34 \mathrm{~nm}$ which correspond to the (002) lattice plane of graphitic carbon. On the other hand, it is observed from Figure $2 \mathrm{~d}$ that the $\mathrm{FeSn}_{2}$ core exhibits a lattice fringe of $d=0.206 \mathrm{~nm}$ which correspond to the (202) lattice plane of FeSn 2 . The OLC shells contain lattice defects, such as carbon layer breakage and blending in the homocentric sphere layers. Moreover, other structural defects, such as interstitial atoms, stacking faults, and dislocation of $C$ layers are also observed [25]. The disordered OLC shells have exposed lattice defects which facilitate the interaction of the $\mathrm{Li}^{+}$ions through them, thereby enhancing the specific discharge capacity and rate capability of the electrocatalytic $\mathrm{FeSn}_{2} / \mathrm{OLC}$ nanocapsules.

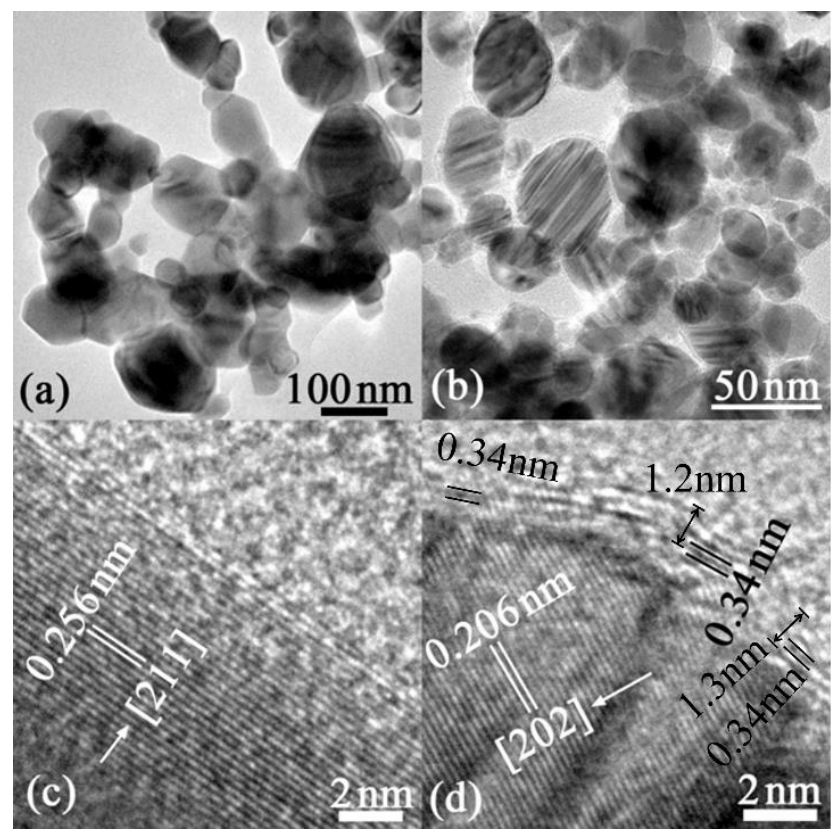

Figure 2. Transmission Electron Microscope (TEM) images of (a) $\mathrm{FeSn}_{2}$ nanoparticles and (b) $\mathrm{FeSn}_{2} / \mathrm{OLC}$ nanocapsules. HR-TEM images of (c) $\mathrm{FeSn}_{2}$ nanoparticles and (d) $\mathrm{FeSn}_{2} / \mathrm{OLC}$ nanocapsules. 


\subsection{Proposed Formation Mechanism of FeSn $n_{2} / \mathrm{OLC}$ Nanocapsules}

From the observations of the physicochemical results of the $\mathrm{FeSn}_{2} / \mathrm{OLC}$ nanocapsules, we have theoretically proposed the formation mechanism of $\mathrm{FeSn}_{2} / \mathrm{OLC}$ nanocapsules during the arc-discharge process. The arc-discharge method scores over the conventional thermal evaporation method in terms of its capability to produce supersaturated atomic hydrogen. The hydrogen not only provided the required heat, but also enabled the formation of tiny bubbles in the molten Fe-Sn alloy. Considering the low inner pressure of the bubbles, they could be approximated as evaporating source in the vacuum. When the surrounding bulk melts and evaporates from the bubble boundary, the bubbles assume an oversaturated state. Finally, the over-saturated vapors escape from the surface of the Fe-Sn molten alloy to form intermetallic nanoparticles. Since the evaporation rates of Fe and Sn at $1873 \mathrm{~K}$ are 1.61 and $47.69 \mathrm{~g} \cdot \mathrm{cm}^{-2} \cdot \mathrm{h}$, respectively, the Sn content in the nanoparticles is much higher than the Fe [26].

The gas phase nucleation during the formation of $\mathrm{FeSn}_{2}$ nanoparticles generally can be classified into three steps as shown in Scheme 1. The driving force and nucleation energy during the process are provided by super cooling which drives the progress of atoms to clusters to nuclei through random collisions [27]. Since the boiling point of Fe and Sn are 3153 and $2876 \mathrm{~K}$, respectively, therefore, under the high thermal energy provided by the hydrogen gas during arc-discharge method, the Sn will evaporate first and form a gas state around the Fe nuclei [26]. In the second step, the Fe nuclei at the core adsorb the Sn atoms or clusters on their surfaces and progressively coagulate to nanoparticles. At the same time, via the interface reactions and interdiffusion of boundaries, the intermetallic Fe-Sn compound phases are formed [27]. The maternal phase Fe finally is consumed by diffusion, leaving behind excess content of $\mathrm{Sn}$ and the $\mathrm{FeSn}_{2}$ nanoparticles as the dominant phase [26]. Since the FeSn powder ingot is composed of excess of Sn content, therefore the formation of the trimers of $\mathrm{FeSn}_{2}$ intermetallic stannide is favored over $\mathrm{Fe}_{2} \mathrm{Sn}$ and $\mathrm{Fe}_{3}$ [26]. Further, the effective formation heat of $\mathrm{FeSn}_{2}$ phase $\left(-0.087 \mathrm{~kJ} \cdot(\mathrm{mol} \cdot \mathrm{atom})^{-1}\right)$ is lower than $\mathrm{FeSn}\left(-0.082 \mathrm{~kJ} \cdot(\mathrm{mol} \cdot \text { atom })^{-1}\right)$, which encourages the formation of $\mathrm{FeSn}_{2}$ [2].

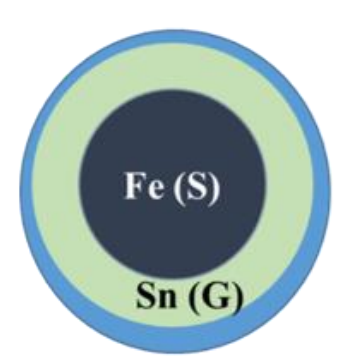

Step 1

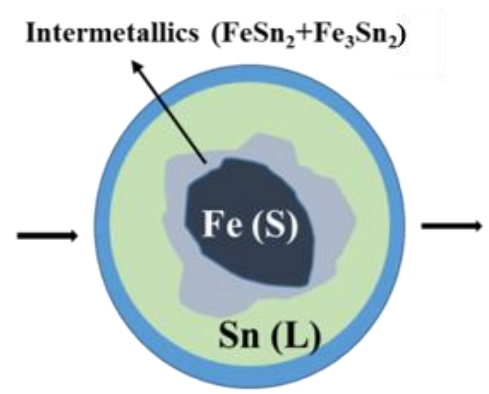

Step 2

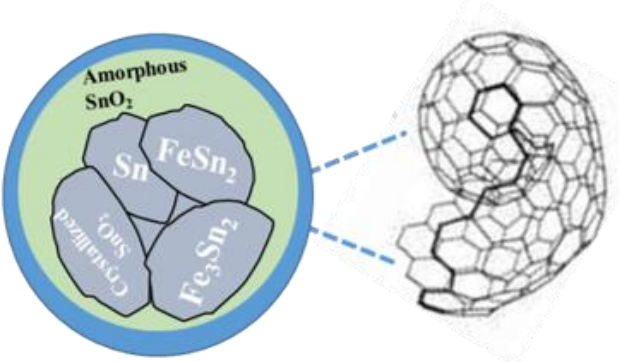

Step 3

OLC shells

Scheme 1. Schematic representation of formation of $\mathrm{FeSn}_{2} / \mathrm{OLC}$ nanocapsules during in-situ arc-discharge process $[20,26]$.

At the very high temperature generated during the arc-discharge method, the ethanol vaporizes to form C2-clusters of carbon. The clusters nucleate to form polycyclic aromatic structures. In order to saturate the bonds, these structures tend to form five-membered rings that create a curvature. A larger number of carbon units get added to expand the ringed structures to onion-like cages. During the arc-discharge, the plasma zone is confined by super-cooling which enables the existing cages to serve as nucleation centers. The growth of the onion-like cages occurs in form of multiple shells. During such a growth, often the inner layers are incomplete before the addition of the next cluster.

Such incomplete layers of carbon onion shells give rise to the defects present in the material. With different degrees of curvature, the proportion of size and number of defects vary [28]. 


\subsection{Electrochemical Performance of LIB Cells}

The electrochemical performance of both the $\mathrm{FeSn}_{2} / \mathrm{OLC}$ nanocapsule-based and the $\mathrm{FeSn}_{2}$ nanoparticle-based electrocatalytic anodes were tested in a LIB half-cell configuration using lithium foils as the cathodes. The voltage versus capacity for the first 3 cycles of $\mathrm{FeSn}_{2} / \mathrm{OLC}$ nanocapsule-based anodes in half-cells are shown in Figure 3a. During the charge-discharge cycles, the following electrocatalytic reactions take place [7]:

$$
\begin{aligned}
& \text { Lithiation: } \mathrm{FeSn}_{2}+8.8 \mathrm{Li}^{+}+8.8 \mathrm{e}^{-} \rightarrow 2 \mathrm{Li}_{4.4} \mathrm{Sn}+\mathrm{Fe} \\
& \text { Delithiation: } 2 \mathrm{Li}_{4.4} \mathrm{Sn}+\mathrm{Fe} \rightarrow 2 \mathrm{Sn}+\mathrm{Fe}+8.8 \mathrm{Li}^{+}+8.8 \mathrm{e}^{-}
\end{aligned}
$$

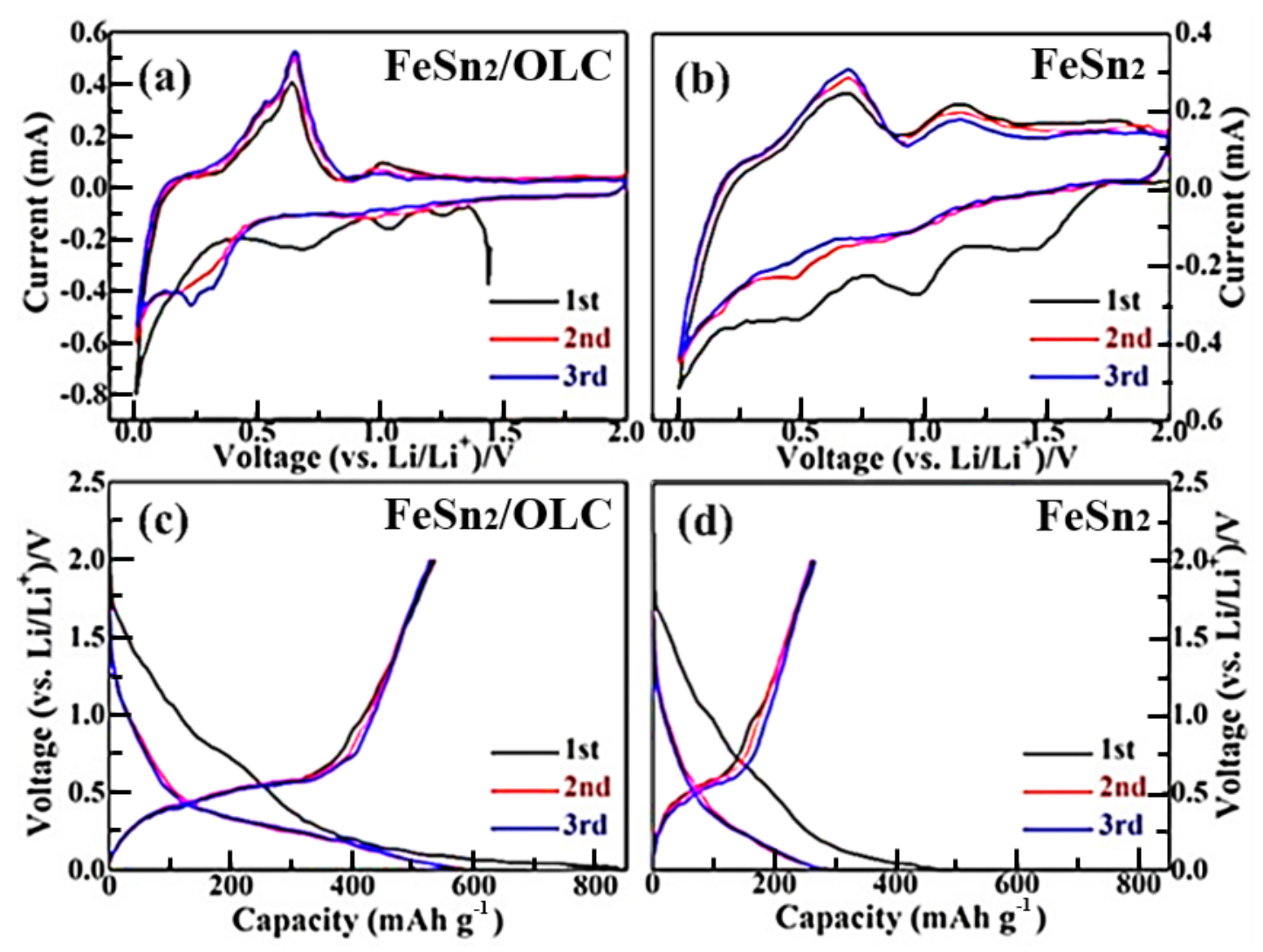

Figure 3. Cyclic voltammetry (CV) curves of LIB cells at the initial three cycles using (a) $\mathrm{FeSn}_{2} / \mathrm{OLC}$ nanocapsule-based anodes and (b) $\mathrm{FeSn}_{2}$ nanoparticle-based anodes. Galvanostatic charge-discharge curves of LIB cells at the initial three cycles using (c) FeSn 2 /OLC nanocapsule-based anodes and (d) $\mathrm{FeSn}_{2}$ nanoparticle-based anodes.

The first cycle exhibits a broad cathodic peak at $0.7 \mathrm{~V}$, while the second and third cycles have multiple cathodic peaks located between the operating voltage window. They are attributed to the lithiation of the tin during the discharge process. Since the potentials of formation of the solid-electrolyte interface (SEI) and lithiation reaction of $\mathrm{FeSn}_{2} / \mathrm{OLC}$ nanocapsule-based anodes in the first discharge process are quite close, hence they overlap to form a broad cathodic peak instead of multiple small

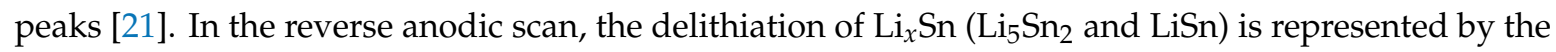
peak at $0.65 \mathrm{~V}$ [2]. The small peak at $\sim 1.0 \mathrm{~V}$ is ascribed to the high surface reactivity for sub- $50 \mathrm{~nm} \mathrm{FeSn_{2 }}$ intermetallic stannide alloy in $\mathrm{FeSn}_{2} / \mathrm{OLC}$ nanocapsules. The large cathodic peak at $0.7 \mathrm{~V}$ is similar for all the three cycles confirming the good reversibility of the reaction and electrochemical stability of the robust $\mathrm{FeSn}_{2} / \mathrm{OLC}$ nanocapsule-based anodes against deformation [7]. However, by the end of the third cycle, the anodic peaks are shifted to high voltages owing to the small polarization arising from 
the increasing impedance of the SEI layer [6]. When compared to the CV curve of $\mathrm{FeSn}_{2}$ in Figure $3 \mathrm{~b}$, it is seen that the oxidation peaks in case of $\mathrm{FeSn}_{2} / \mathrm{OLC}$ nanocapsule-based anodes are sharper than that of the $\mathrm{FeSn}_{2}$ nanoparticle-based anodes. This proves the better electrochemical kinetics of the $\mathrm{FeSn}_{2} / \mathrm{OLC}$ nanocapsules owing to their highly conductive OLC shells. In the subsequent cycles, the intensity and integrated areas of peaks decrease because of the irreversible capacity attenuation.

It is observed from the galvanostatic charge-discharge profile of electrocatalytic $\mathrm{FeSn}_{2} / \mathrm{OLC}$ nanocapsule-based anodes in Figure $3 c$ that in the first discharge curve there is a slight bump at $\sim 0.6$ $\mathrm{V}$ because of the irreversible formation of SEI layer on the surface of the anode [7]. It disappears in the subsequent cycles. The plateau between 0.3 and $0.0 \mathrm{~V}$ corresponds to the lithiation of the anode. In the subsequent cycles this plateau is heavily overlapped signifying their good reversibility and electrochemical stability. Figure $3 \mathrm{~d}$ shows that both $\mathrm{FeSn}_{2}$ nanoparticle-based anodes and $\mathrm{FeSn}_{2} / \mathrm{OLC}$ nanocapsule-based anodes have high specific discharge capacity of 464 and $835 \mathrm{mAh} \cdot \mathrm{g}^{-1}$, respectively. It is noted that the initial specific discharge capacity of the $\mathrm{FeSn}_{2} / \mathrm{OLC}$ nanocapsule-based anodes $\left(835 \mathrm{mAh} \cdot \mathrm{g}^{-1}\right)$ was higher than the theoretical specific discharge capacity of the $\mathrm{FeSn}_{2}$ nanoparticle-based anodes $\left(\sim 804 \mathrm{mAh} \cdot \mathrm{g}^{-1}\right)$ [21]. This is attributed to the following four possibilities: (1) The theoretical capacity of $\mathrm{FeSn}_{2}$ is based on the conventional alloying mechanism, in which the reduction reaction of $\mathrm{FeSn}_{2}$ is assumed to be irreversible [29]; (2) an organic polymeric/gel-like film might had formed at the interface of the core/shell-structured anode, which enhanced their capacity via "pseudo-capacitance" [30]; (3) the hollow structure and large surface area of the OLC shells enabled the storage of $\mathrm{Li}^{+}$ions at the interfaces and pores of the electrocatalytic $\mathrm{FeSn}_{2} / \mathrm{OLC}$ nanocapsule-based anodes [11,19]; and (4) the high electrical conductivity of the OLC shell facilitated electron transfer during lithiation and delithiation process [20,31]. On the other hand, the poor electrocatalytic performance of the $\mathrm{FeSn}_{2}$ nanoparticle-based anodes is rooted in their electrochemical instability, since the $\mathrm{FeSn}_{2}$ nanoparticles undergo pulverization and exfoliation during lithiation-delithiation process $[2,10,11]$. The initial columbic efficiency of $\mathrm{FeSn}_{2} / \mathrm{OLC}$ nanocapsule-based anodes was $\sim 64.2 \%$, while it was only $57.1 \%$ in the FeSn 2 nanoparticle-based anodes. It can be concluded that the high specific discharge capacity, relatively flat plateaus with minimal hysteresis loss, cyclic stability, and maximized cell voltage at low reaction potential are attributed to the improved electrocatalytic properties of $\mathrm{FeSn}_{2} / \mathrm{OLC}$ nanocapsules, thus making them promising anodes for LIBs. Additionally, the low reaction potential (higher than graphite) of $\mathrm{FeSn}_{2} / \mathrm{OLC}$ nanocapsule-based anodes would allow a degree of safety when related to lithium plating [32].

The cycling performance of LIBs is directly proportional to the electrochemical stability of their electrocatalytic anodes. The cycling performance of LIB cells composed of the $\mathrm{FeSn}_{2} / \mathrm{OLC}$ nanocapsule-based anodes and the $\mathrm{FeSn}_{2}$ nanoparticle-based anodes at a specific current density of $50 \mathrm{~mA} \cdot \mathrm{g}^{-1}$ is shown in Figure $4 \mathrm{a}$. At the end of 100 charge-discharge cycles, the FeSn ${ }_{2}$ nanoparticle-based anodes only show a specific discharge capacity of $\sim 178 \mathrm{mAh} \cdot \mathrm{g}^{-1}(\sim 38.6 \%$ retention of the initial specific discharge capacity) compared to $\sim 519 \mathrm{mAh} \cdot \mathrm{g}^{-1}$ ( $\sim 62.1 \%$ retention of initial specific discharge capacity) in the $\mathrm{FeSn}_{2} / \mathrm{OLC}$ nanocapsule-based anodes. The cycling instability of the FeSn 2 nanoparticle-based anodes can be attributed to the irreversible formation of $\mathrm{Li}_{2} \mathrm{O}$ matrix, active retention of $\mathrm{Li}-\mathrm{Sn}$ compounds, and continuous consumption of $\mathrm{Li}^{+}$ions during formation and decomposition of SEI films $[7,12]$. On the other hand, the $\mathrm{FeSn}_{2} / \mathrm{OLC}$ nanocapsule-based anodes show a reduced capacity fading to as low as $0.3 \%$ per cycle. The improvement in surface area, protection provided by the OLC shells to the FeSn 2 cores, and efficient electron transfer across the conductive OLC shells [33] are the primary reasons for the improved performance. The OLC shells protected the core FeSn 2 nanoparticles from coming in direct contact with the electrolyte and prevented the formation of SEI on them, eventually delaying the deactivation. With increasing cycle number, the specific capacity of the LIB cells with $\mathrm{FeSn}_{2} / \mathrm{OLC}$ nanocapsule-based anode gradually decreased till the 70th cycle. After that a slight rise in capacity was observed. The elevation in capacity can be ascribed to the delayed activation of the anodes [34]. Such an activation period was absent in $\mathrm{FeSn}_{2}$ nanoparticle-based anode. Though the initial coulombic efficiency of the $\mathrm{FeSn}_{2} / \mathrm{OLC}$ nanocapsule-based anode was only $64.2 \%$, 
but it increased to $92.5 \%$ in the third cycle and was maintained till 100 cycles indicating the highly reversible lithium insertion/extraction process during the fast electron transport within the electrodes and their electrochemical stability. The initial drop in coulombic efficiency was due to the irreversible processes that rendered some of the electrocatalytic active sites as inactive [30].
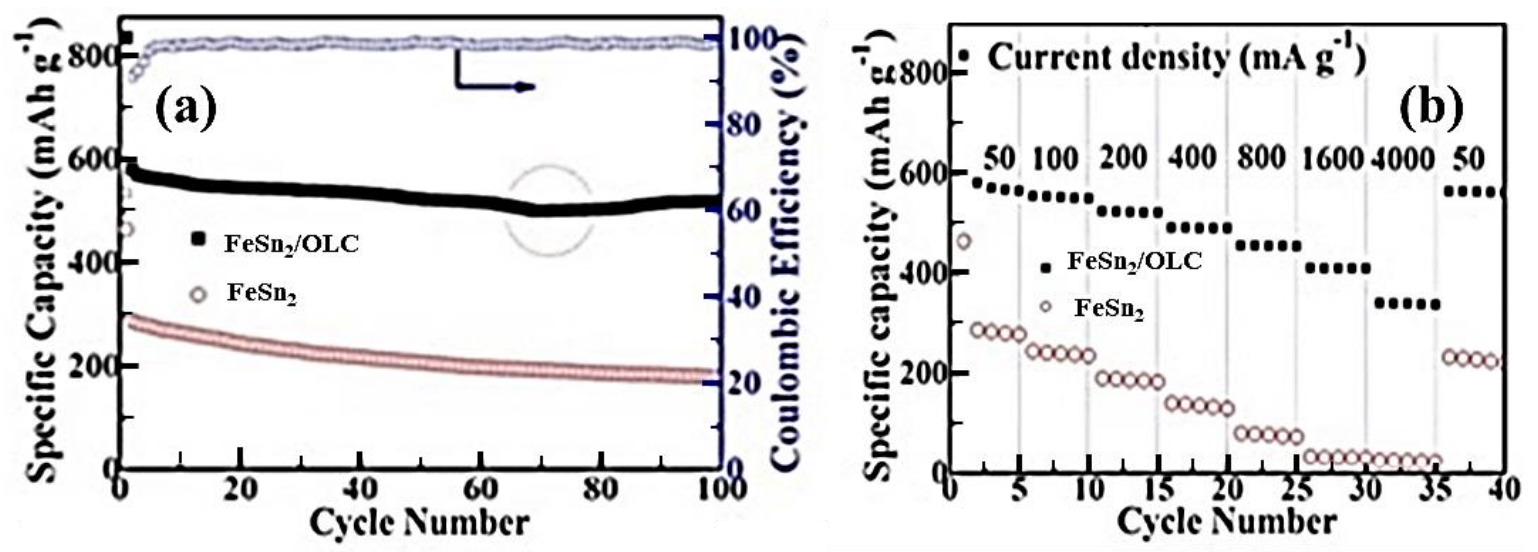

Figure 4. (a) Cyclic stability of LIB cells at a specific current density of $50 \mathrm{~mA} \cdot \mathrm{g}^{-1}$ using FeSn $/ \mathrm{OLC}$ nanocapsule-based anodes and $\mathrm{FeSn}_{2}$ nanoparticle-based anodes. (b) Rate of performance of $\mathrm{FeSn}_{2} / \mathrm{OLC}$ nanocapsule-based anodes and $\mathrm{FeSn}_{2}$ nanoparticle-based anodes.

The LIB cells with the FeSn $/$ /OLC nanocapsule-based anodes exhibit an improved rate capability than the $\mathrm{FeSn}_{2}$ nanoparticle-based anodes. It is observed from Figure $4 \mathrm{~b}$ that the $\mathrm{FeSn} 2$ nanoparticle-based anodes show an initial specific discharge capacity of $464 \mathrm{mAh} \cdot \mathrm{g}^{-1}$ at a specific current density of $50 \mathrm{~mA} \cdot \mathrm{g}^{-1}$. However, at a higher specific current density of $4000 \mathrm{mAh} \cdot \mathrm{g}^{-1}$, the specific discharge capacity is reduced to $22 \mathrm{mAh} \cdot \mathrm{g}^{-1}$ ( 4.7\% specific discharge capacity retention). When the specific current density is returned to $50 \mathrm{~mA} \cdot \mathrm{g}^{-1}$, the specific discharge capacity recovers only till $232 \mathrm{mAh} \cdot \mathrm{g}^{-1}$. The high loss in specific discharge capacity retention for the $\mathrm{FeSn}_{2}$ nanoparticle-based anodes is due to the giant volume changes suffered by the $\mathrm{FeSn}_{2}$ nanoparticles during lithium insertion and removal. This leads to particle fragmentation, loss of electrical contacts, and capacity fading of the LIB cells. In contrast, the FeSn 2 /OLC nanocapsule-based anodes deliver enhanced specific discharge capacities of 836 and $340 \mathrm{mAh} \cdot \mathrm{g}^{-1}$ at 50 and $4000 \mathrm{~mA} \cdot \mathrm{g}^{-1}$ specific current density, respectively. When the specific current density is returned to $50 \mathrm{~mA} \cdot \mathrm{g}^{-1}$, the specific discharge capacity is enhanced to $564 \mathrm{mAh} \cdot \mathrm{g}^{-1}$, indicating $67.5 \%$ specific discharge capacity retention as compared to only $50 \%$ in the $\mathrm{FeSn}_{2}$ nanoparticle-based anodes. In the second cycle, at $50 \mathrm{~mA} \cdot \mathrm{g}^{-1}$, the specific discharge capacity retention in the $\mathrm{FeSn}_{2} / \mathrm{OLC}$ nanocapsule-based anodes is $\sim 97.2 \%$. The excellent electrochemical stability and rate performance of the $\mathrm{FeSn}_{2} / \mathrm{OLC}$ nanocapsule-based anodes is due to the small particle size which allow rapid mass transfer between the electrode and electrolytes $[10,11]$. Further, the OLC shells allow the space required for the volume expansion during the lithiation-delithiation process $[2,19,35]$.

As shown in Figure 5, the EIS was carried out to investigate the electrochemical properties of the active materials: $\mathrm{FeSn}_{2} / \mathrm{OLC}$ nanocapsule-based anodes and $\mathrm{FeSn}_{2}$ nanoparticle-based anodes in LIB cells. Subsequently, the Nyquist plots were fitted with ZView software. The equivalent circuit model was composed of the following parameters: $R_{\mathrm{S}}$ (ohmic resistance), $R_{\mathrm{ct}}$ (interface charge transfer resistance), CPE (constant phase element), and $Z_{\mathrm{W}}$ (Warburg impedance). The high frequency intercept on the real axis represents $R_{\mathrm{s}}$ arising from the electrolyte and electrode resistances. $R_{\mathrm{ct}}$ is the resistance during transfer of electrons from electrode to electrolyte. $\mathrm{CPE}$ is attributed to the double layer capacitance imparted by the SEI layer. $Z_{\mathrm{w}}$ represents the finite diffusion of the electrons through the anode [36,37]. Owing to increased electrical conductivity and reduced volume expansion in the $\mathrm{FeSn}_{2} / \mathrm{OLC}$ nanocapsule-based anodes, they feature a lower $R_{\mathrm{s}}$ than the $\mathrm{FeSn}_{2}$ nanoparticle-based anodes, both before and after cycling [33]. The lower $R_{\mathrm{ct}}$ of the $\mathrm{FeSn}_{2} / \mathrm{OLC}$ nanocapsule-based 
anodes facilitates faster $\mathrm{Li}^{+}$ions diffusion and electron transfer, consequently enhancing the cycling performance even at high current densities.

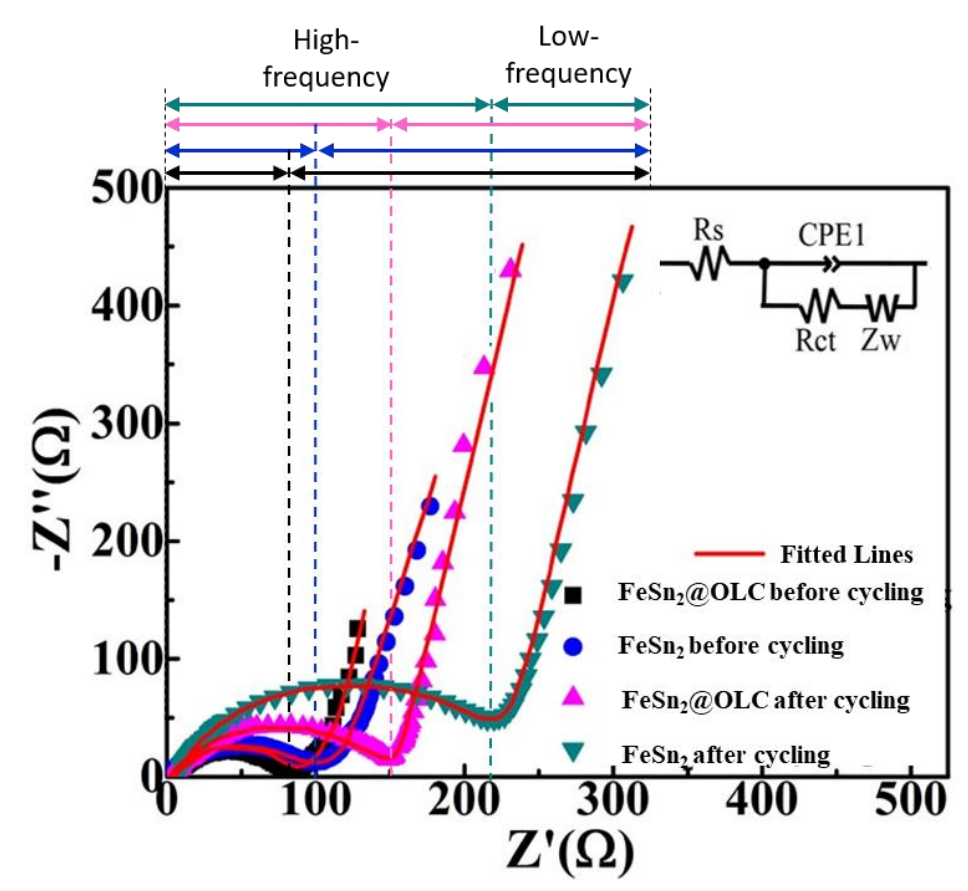

Figure 5. Electrochemical impedance spectra (EIS) of LIB cells using FeSn $2 / O L C$ nanocapsule-based anodes and $\mathrm{FeSn}_{2}$ nanoparticle-based anodes. The inset shows the equivalent circuit used for fitting the experimental EIS data.

Overall, the electrocatalytic performance and electrochemical stability of the $\mathrm{FeSn}_{2} / \mathrm{OLC}$ nanocapsule-based anodes improve because of the following factors: (i) sub-50 nm pure phase crystalline $\mathrm{FeSn}_{2}$ nanoparticle as cores provide high storage and specific discharge capacity by combining with $\mathrm{Li}^{+}$ions; (ii) $\mathrm{Fe}$ act as buffering agents and facilitate the even redistribution of the internal stress during lithiation-delithation, thereby preventing/delaying the pulverization and aggregation of tin; (iii) the OLC shell impart higher electrical conductivity, suppress the FeSn 2 crystal size, accommodate the strains during lithiation-induced volume change, and protect the $\mathrm{FeSn}_{2}$ from direct contact of electrolyte and subsequent formation of SEI.

\section{Materials and Methods}

\subsection{Materials}

Metallic powders of iron and tin of $99.9 \%$ purity with an average size of $5 \mu \mathrm{m}$, graphite needle, absolute ethanol, carbon black, PVDF (polyvinylidene fluoride), NMP ( $N$-methyl pyrrolidone), copper foils, lithium foils, polypropylene (PP) film, $1 \mathrm{M} \mathrm{LiPF}_{6}$ in ethylene carbonate (EC)/diethyl carbonate (DEC) (1:1 in volume), were purchased from Acros Organics (Geel 2440, Belgium) and used without any further purification, unless otherwise stated.

\subsection{Synthesis of $\mathrm{FeSn}_{2} / \mathrm{OLC}$ Nanocapsules}

The schematic of in-situ arc-discharge process for synthesis of $\mathrm{FeSn}_{2} / \mathrm{OLC}$ nanocapsules is shown in Scheme 2. Briefly, iron and tin powders were mixed well to prepare a $\mathrm{Fe}_{95} \mathrm{Sn}_{5}$ powder mixture. The powder mixture was further subject to $20 \mathrm{MPa}$ pressure to form $20 \mathrm{~mm}$ cylindrical and compact FeSn powder ingots. During the arc-discharge process, the FeSn powder ingot was used as an anode while the graphite needle served as the cathode. The chamber (Mianyang 510700, Sichuan, China) was evacuated to maintain vacuum pressure at $6 \mathrm{mPa}$. Along with pure argon at $20 \mathrm{kPa}$ and hydrogen at 
$10 \mathrm{kPa}, 30 \mathrm{~mL}$ of ethanol was injected into the chamber. This liquid ethanol served as the source of carbon which constituted the defective onion-like carbon shells. On entering the high temperature arc-discharge chamber, the ethanol was dissociated to $\mathrm{C}, \mathrm{H}$, and $\mathrm{O}$. On the other hand, the argon produced the argon-plasma, while hydrogen aided the evaporation of Fe and $\mathrm{Sn}$ from the FeSn powder ingot. The ingot was evaporated in 10-15 min by arc-discharging at $\sim 40 \mathrm{~V}$ and $\sim 20 \mathrm{~A}$. The current and voltage conditions during the process were automatically adjusted by the distance between the electrodes. To prevent any serious explosion from the highly reactive products, they were passivated with argon for $12 \mathrm{~h}$ and finally collected from the top of the chamber.

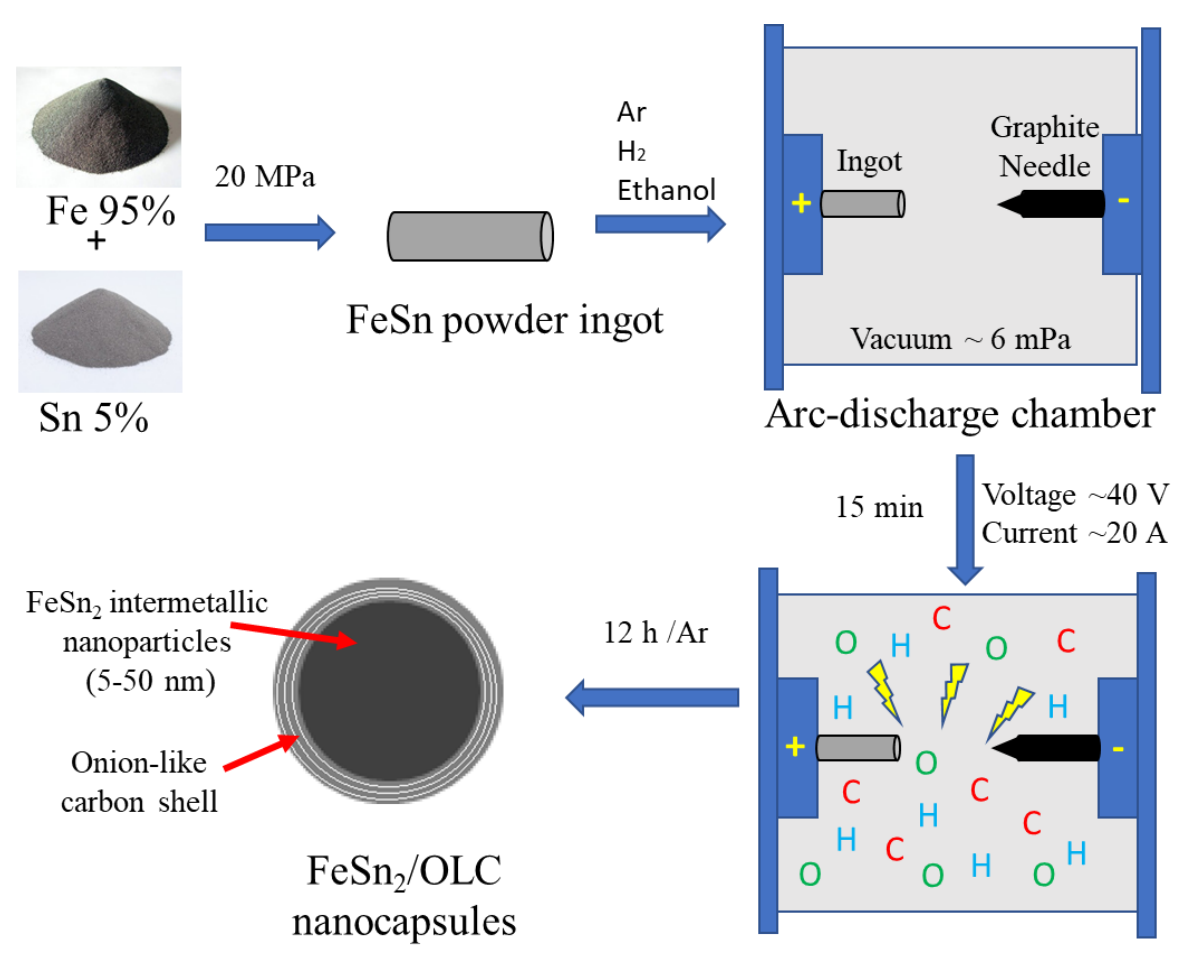

Scheme 2. Schematic of in-situ arc-discharge process to synthesize $\mathrm{FeSn}_{2} / \mathrm{OLC}$ nanocapsules.

\subsection{Physicochemical Characterizations of FeSn $n_{2} / \mathrm{OLC}$ Nanocapsules}

The phase constituency of the in-situ arc-discharge-derived $\mathrm{FeSn}_{2} / \mathrm{OLC}$ stannide-based nanocapsules was characterized using a Bruker D8 Advance X-ray diffractometer (XRD, Karlsruhe 76187, Germany) with a monochromatized $\mathrm{Cu}-\mathrm{K} \alpha$ radiation and operated at a voltage of $30 \mathrm{kV}$, a current of $30 \mathrm{~mA}$, and a scan step of $0.2^{\circ}$. The surface morphology and internal structure of the $\mathrm{FeSn}_{2} / \mathrm{OLC}$ nanocapsules were evaluated using a JEOL 2010 transmission electron microscope (TEM, Tokyo 196-8558, Japan) at an accelerating voltage of $200 \mathrm{kV}$.

\subsection{Preparation of FeSn $/ \mathrm{OLC}$ Nanocapsules as Stannide-Based Electrocatalytic Anodes and Fabrication of Their LIB Cells}

The $\mathrm{FeSn}_{2} / \mathrm{OLC}$ nanocapsule-based electrocatalytic anodes were prepared by making a homogeneous slurry composed of $80 \mathrm{wt} . \% \mathrm{FeSn}_{2} / \mathrm{OLC}$ nanocapsules, $10 \mathrm{wt}$ \% carbon black as the conducting agent and $10 \mathrm{wt} . \%$ PVDF binder dissolved in NMP. The slurry was coated on copper foils and finally heated under vacuum at $120{ }^{\circ} \mathrm{C}$ for $24 \mathrm{~h}$. As a control for comparison, pure $\mathrm{FeSn}_{2}$ nanoparticle anode was also prepared in the similar way.

The LIB cells were assembled in an argon filled glovebox (Vacuum Technology Inc., Oak Ridge 37830, United States) to avoid contamination. Briefly, lithium foils as the cathode, PP film as the separator, $1 \mathrm{M} \mathrm{LiPF}_{6}$ in EC/ DEC (1:1 in volume) as the electrolyte, and the slurry-coated copper foils as the anode were assembled into a coin cell 2025. 


\subsection{Electrochemical Characterizations of LIB Cells}

The two-electrode system LIB cells were electrochemically tested with an electrochemical impedance spectroscope (EIS) (CHI 660E, Shanghai 201500, China). The cyclic voltammetry (CV) and Nyquist measurements were taken before and after the cycles. The CV was conducted at a voltage sweep rate of $0.1 \mathrm{mV} \cdot \mathrm{s}^{-1}$ and a frequency range of $10 \mathrm{mHz}-100 \mathrm{kHz}$. The impedance spectra were recorded at a perturbation amplitude of $5 \mathrm{mV}$. The galvanostatic measurements were carried out in the battery testing system (Lanhe CT 2001, Wuhan 430014, China) within a voltage range of 0.05-2.0 V (vs. $\mathrm{Li} / \mathrm{Li}^{+}$) and specific current density of $50 \mathrm{~mA} \cdot \mathrm{g}^{-1}$. The rate capabilities of the electrodes were measured by cycling the LIB cells between 0.05 and $2.0 \mathrm{~V}$ at different specific current densities from 50 to $4000 \mathrm{~mA} \cdot \mathrm{g}^{-1}$ and back to $50 \mathrm{~mA} \cdot \mathrm{g}^{-1}$. At each of the specific current density, five cycles were recorded.

\section{Conclusions}

In this work, a facile one-pot in-situ arc-discharge method has been successfully used to synthesize core/shell-structured $\mathrm{FeSn}_{2} / \mathrm{OLC}$ nanocapsules, which act as a stable and high-performance stannide-based electrocatalytic anode material for LIBs. The arc-discharge process has enabled in assembling the $\mathrm{FeSn}_{2} / \mathrm{OLC}$ nanocapsules through an in situ method, by confining the $\mathrm{FeSn}_{2}$ crystal size to sub-50 nm, introducing an intermetallic stannide alloy, and combining it with a conductive onion-like carbon shell. The $\mathrm{FeSn}_{2} / \mathrm{OLC}$ nanocapsule-based anodes have exhibited improved electrocatalytic properties such as high reversible capacity, good cyclability, and superior rate capability when compared to their $\mathrm{FeSn}_{2}$ nanoparticle-based anodes counterpart. This is attributed to the electrocatalytic $\mathrm{FeSn}_{2}$ nanoparticle cores in the $\mathrm{FeSn}_{2} / \mathrm{OLC}$ nanocapsule anodes which effectively enable a reversible lithiation process during charge-discharge of the LIB cells, while the OLC shells enhance electrochemical stability by imparting high electrical conductivity and protecting the $\mathrm{FeSn}_{2}$ nanoparticle cores from volume change-induced pulverization and solid electrolyte interphase-induced passivation. The OLC shells also confine the growth of $\mathrm{FeSn}_{2}$ nanoparticles to 5-50 $\mathrm{nm}$ size, thereby improving the electrochemical kinetics and surface contact area of the electrodes and electrolyte. Therefore, the resistance offered by our proposed $\mathrm{FeSn}_{2} / \mathrm{OLC}$ nanocapsule-based anodes is much smaller in comparison with the $\mathrm{FeSn}_{2}$ nanoparticle-based anodes. The presence of the OLC shells have overcome the most important problem of LIBs, that is, the deterioration of electrocatalytic anodes because of the volume changes during lithiation and delithiation process. The OLC shells have allowed the space for expansion during lithiation and buffered the stress induced thereby. Therefore, the $\mathrm{FeSn}_{2} / \mathrm{OLC}$ nanocapsule-based anodes have shown high specific discharge capacity retention even after 100 cycles, thus proving their high electrochemical stability. It can be concluded that the arc-discharge method for synthesizing high-performance electrocatalytic $\mathrm{FeSn}_{2} / \mathrm{OLC}$ nanocapsule-like anodes stand out in comparison to other reported methods such as solvothermal, chemical reduction, and ball-milling [38]. In case of solvothermal synthesis, the control of nanoparticles size and morphology requires complicated and multiple steps and huge quantities of waste solvent recycling. On the other hand, the chemical reduction process leads to aggregated nanoparticles [29]. The ball-milling technique suffers from lack of desired control on the phase and structure of the nanomaterials [26]. Arc-discharge method not only alleviates the above mentioned problems, but it is also economical, environmentally benign, facile, practically zero-waste, and scalable process $[10,11,39]$. This method can be extended to develop other core/shell-structured electrocatalytic materials with controlled morphology for applications in batteries, supercapacitors, and fuel cells.

Author Contributions: D.H. and A.C. conducted the experiments, analyzed the results, and wrote up the manuscript. L.H.M. assisted in analyzing the results and writing up the manuscript. S.W.O. conceived the projects and guided D.H., A.C., and L.H.M. in the whole work.

Funding: Research Grants Council of the HKSAR Government (Grant Nos. R5020-18 and 15217917) and Innovation and Technology Commission of the HKSAR Government to the Hong Kong Branch of National Rail Transit Electrification and Automation Engineering Technology Research Center (Grant No. K-BBY1). 
Acknowledgments: This work was supported by the Research Grants Council of the HKSAR Government (R5020-18 and 15217917) and the Innovation and Technology Commission of the HKSAR Government to the Hong Kong Branch of National Rail Transit Electrification and Automation Engineering Technology Research Center (K-BBY1).

Conflicts of Interest: The authors declare no conflict of interest. The funders had no role in the design of the study; in the collection, analyses, or interpretation of data; in the writing of the manuscript, and in the decision to publish the results.

\section{References}

1. Zhou, R.; Wang, J.G.; Liu, H.; Liu, H.; Jin, D.; Liu, X.; Shen, C.; Xie, K.; Wei, B. Coaxial MoS2@Carbon hybrid fibers: A low-cost anode material for high-performance Li-ion batteries. Materals 2017, 10, 174. [CrossRef]

2. Liu, C.J.; Xue, F.H.; Huang, H.; Yu, X.H.; Xie, C.J.; Shi, M.S.; Cao, G.Z.; Jung, Y.G.; Dong, X.L. Preparation and Electrochemical properties of Fe-Sn (C) Nanocomposites as Anode for Lithium-ion Batteries. Electrochim. Acta 2014, 129, 93-99. [CrossRef]

3. Diouf, B.; Pode, R. Potential of lithium-ion batteries in renewable energy. Renew. Energy 2015, 76, 375-380. [CrossRef]

4. Lu, L.; Han, X.; Li, J.; Hua, J.; Ouyang, M. A review on the key issues for lithium-ion battery management in electric vehicles. J. Power Sources 2013, 226, 272-288. [CrossRef]

5. Chen, J. Recent Progress in Advanced Materials for Lithium Ion Batteries. Materals 2013, 6, $156-183$. [CrossRef]

6. Wan, Y.; Liu, J.; Liu, C.; Ji, S.; Zhou, Y. Facile Synthesis of FeSn 2 Alloy Nanoparticles as Anode Materials for Lithium-Ion Batteries. Energy Environ. Focus 2013, 2, 63-67. [CrossRef]

7. Leibowitz, J.; Allcorn, E.; Manthiram, A. FeSn 2 -TiC nanocomposite alloy anodes for lithium ion batteries. J. Power Sources 2015, 295, 125-130. [CrossRef]

8. Qin, J.; Liu, D.; Zhang, X.; Zhao, N.; Shi, C.; Liu, E.; He, F.; Ma, L.; Li, Q.; Li, J.; et al. One-step synthesis of SnCo nanoconfined in hierarchical carbon nanostructures for lithium ion battery anode. Nanoscale 2017, 9, 15856-15864. [CrossRef]

9. Lee, J.M.; Chang, W.S.; Yu, B.C.; Kim, H.; Im, D.; Doo, S.G.; Sohn, H.J. Enhancement of cyclability using recombination reaction of $\mathrm{Cu}$ for $\mathrm{Sn}_{2} \mathrm{Fe}$ nanocomposite anode for lithium-ion batteries. Electrochem. Commun. 2010, 12, 928-932. [CrossRef]

10. Liu, X.; Or, S.W.; Jin, C.; Lv, Y.; Feng, C.; Sun, Y. NiO/C nanocapsules with onion-like carbon shell as anode material for lithium ion batteries. Carbon 2013, 60, 215-220. [CrossRef]

11. Han, D.; Song, G.; Liu, B.; Yan, H. Core-shell-structured nickel ferrite/onion-like carbon nanocapsules: An anode material with enhanced electrochemical performance for lithium-ion batteries. RSC Adv. 2015, 5, 42875-42880. [CrossRef]

12. Kravchyk, K.; Protesescu, L.; Bodnarchuk, M.I.; Krumeich, F.; Yarema, M.; Walter, M.; Guntlin, C.; Kovalenko, M.V. Monodisperse and inorganically capped $\mathrm{Sn}$ and $\mathrm{Sn} / \mathrm{SnO}_{2}$ nanocrystals for high-performance Li-ion battery anodes. J. Am. Chem. Soc. 2013, 135, 4199-4202. [CrossRef]

13. Li, Q.-Y.; Pan, Q.-C.; Yang, G.-H.; Lin, X.-L.; Yan, Z.-X.; Wang, H.-Q.; Huang, Y.-G. Synthesis of Sn/MoS2/C composites as high-performance anodes for lithium-ion batteries. J. Mater. Chem. A 2015, 3, 20375-20381. [CrossRef]

14. Ferrara, G.; Damen, L.; Arbizzani, C.; Inguanta, R.; Piazza, S.; Sunseri, C.; Mastragostino, M. SnCo nanowire array as negative electrode for lithium-ion batteries. J. Power Sources 2011, 196, 1469-1473. [CrossRef]

15. Chamas, M.; Lippens, P.E.; Jumas, J.C.; Hassoun, J.; Panero, S.; Scrosati, B. Electrochemical impedance characterization of $\mathrm{FeSn}_{2}$ electrodes for Li-ion batteries. Electrochim. Acta 2011, 56, 6732-6736. [CrossRef]

16. Armbrüster, M.; Schnelle, W.; Cardoso-Gil, R.; Grin, Y. Chemical bonding in compounds of the $\mathrm{CuAl}_{2} \mathrm{family:}$ $\mathrm{MnSn}_{2}, \mathrm{FeSn}_{2}$ and $\mathrm{CoSn}_{2}$. Chem. A Eur. J. 2010, 16, 10357-10365. [CrossRef]

17. Han, D.; Or, S.W.; Dong, X.; Liu, B. FeSn 2 /defective onion-like carbon core-shell structured nanocapsules for high-frequency microwave absorption. J. Alloys Compd. 2017, 695, 2605-2611. [CrossRef]

18. Pech, D.; Brunet, M.; Durou, H.; Huang, P.; Mochalin, V.; Gogotsi, Y.; Taberna, P.L.; Simon, P. Ultrahigh-power micrometre-sized supercapacitors based on onion-like carbon. Nat. Nanotechnol. 2010, 5, 651-654. [CrossRef]

19. Zeiger, M.; Jäckel, N.; Mochalin, V.N.; Presser, V. Review: Carbon onions for electrochemical energy storage. J. Mater. Chem. A 2016, 4, 3172-3196. [CrossRef] 
20. Van Aken, K.L.; Maleski, K.; Mathis, T.S.; Breslin, J.P.; Gogotsi, Y. Processing of Onion-like Carbon for Electrochemical Capacitors. ECS J. Solid State Sci. Technol. 2017, 6, M3103-M3108. [CrossRef]

21. Wang, S.; He, M.; Walter, M.; Krumeich, F.; Kravchyk, K.V.; Kovalenko, M.V. Monodisperse CoSn2and FeSn2nanocrystals as high-performance anode materials for lithium-ion batteries. Nanoscale 2018, 10, 6827-6831. [CrossRef]

22. Armbruster, M.; Schmidt, M.; Cardoso-Gil, R.; Borrmann, H.; Grin, Y. Crystal structures of iron distannide, $\mathrm{FeSn}_{2}$, and cobalt distannide, $\mathrm{CoSn}_{2}$. Z. fur Krist.-New Cryst. Struct. 2007, 222, 83-84. [CrossRef]

23. Nwokeke, U.G.; Alcántara, R.; Tirado, J.L.; Stoyanova, R.; Yoncheva, M.; Zhecheva, E. Electron paramagnetic resonance, X-ray diffraction, mössbauer spectroscopy, and electrochemical studies on nanocrystalline $\mathrm{FeSn}_{2}$ obtained by reduction of salts in tetraethylene glycol. Chem. Mater. 2010, 22, 2268-2275. [CrossRef]

24. Liu, X.; Wu, N.; Cui, C.; Zhou, P.; Sun, Y. Enhanced rate capability and cycling stability of core/shell structured $\mathrm{CoFe}_{2} \mathrm{O}_{4}$ /onion-like $\mathrm{C}$ nanocapsules for lithium-ion battery anodes. J. Alloys Compd. 2015, 644, 59-65. [CrossRef]

25. Jiang, L.; Wang, Z.; Geng, D.; Lin, Y.; Wang, Y.; An, J.; He, J.; Li, D.; Liu, W.; Zhang, Z. Structure and electromagnetic properties of both regular and defective onion-like carbon nanoparticles. Carbon 2015, 95, 910-918. [CrossRef]

26. Lei, J.P.; Dong, X.L.; Zhu, X.G.; Lei, M.K.; Huang, H.; Zhang, X.F.; Lu, B.; Park, W.J.; Chung, H.S. Formation and characterization of intermetallic Fe-Sn nanoparticles synthesized by an arc discharge method. Intermetallics 2007, 15, 1589-1594. [CrossRef]

27. Thompson, C.V. On the role of diffusion in phase selection during reactions at interfaces. J. Mater. Res. 1992, 7,367-373. [CrossRef]

28. Growth Mechanisms of Carbon Onions Obtained by Thermal Treatment - Diamond Films. Texas Powerful Smart. 2019. Available online: https://www.texaspowerfulsmart.com/diamond-films/growth-mechanismsof-carbon-onions-obtained-by-thermal-treatment.html (accessed on 7 November 2019).

29. Zhang, C.Q.; Tu, J.P.; Huang, X.H.; Yuan, Y.F.; Wang, S.F.; Mao, F. Preparation an electrochemical performances of nanoscale FeSn 2 as anode material for lithium ion batteries. J. Alloys Compd. 2008, 457, 81-85. [CrossRef]

30. Yuan, Y.; Amine, K.; Lu, J.; Shahbazian-Yassar, R. Understanding materials challenges for rechargeable ion batteries with in situ transmission electron microscopy. Nat. Commun. 2017, 8, 15806. [CrossRef]

31. Liu, Y.; Kim, D.Y. Enhancement of capacitance by electrochemical oxidation of nanodiamond derived carbon nano-onions. Electrochim. Acta 2014, 139, 82-87. [CrossRef]

32. An, S.J.; Li, J.; Daniel, C.; Mohanty, D.; Nagpure, S.; Wood, D.L. The state of understanding of the lithium-ion-battery graphite solid electrolyte interphase (SEI) and its relationship to formation cycling. Carbon 2016, 105, 52-76. [CrossRef]

33. Liu, X.; Wu, Y.; Li, X.; Yu, J.; Sun, Y. FeS@onion-like carbon nanocapsules embedded in amorphous carbon for the lithium ion batteries with excellent cycling stability. Ceram. Int. 2018, 44, 13654-13661. [CrossRef]

34. Yabuuchi, N.; Kubota, K.; Aoki, Y.; Komaba, S. Understanding Particle-Size-Dependent Electrochemical Properties of $\mathrm{Li}_{2} \mathrm{MnO}_{3}$-Based Positive Electrode Materials for Rechargeable Lithium Batteries. J. Phys. Chem. C 2016, 120, 875-885. [CrossRef]

35. Li, X.; He, X.; Xu, Y.; Huang, L.; Li, J.; Sun, S.; Zhao, J. Superiority of the bi-phasic mixture of a tin-based alloy nanocomposite as the anode for lithium ion batteries. J. Mater. Chem. A 2015, 3, 3794-3800. [CrossRef]

36. Osaka, T.; Mukoyama, D.; Nara, H. Review_Development of Diagnostic Process for Commercially Available Batteries, Especially Lithium Ion Battery, by Electrochemical Impedance Spectroscopy. J. Electrochem. Soc. 2015, 162, A2529-A2537. [CrossRef]

37. Qin, F.; Zhang, K.; Fang, J.; Lai, Y.; Li, Q.; Zhang, Z.; Li, J. High performance lithium sulfur batteries with a cassava-derived carbon sheet as a polysulfides inhibitor. New J. Chem. 2014, 38, 4549-4554. [CrossRef]

38. Kwon, Y.S.; Choi, P.P.; Kim, J.S.; Kwon, D.H.; Gerasimov, K.B. Decomposition of intermetallics during high-energy ball-milling. Mater. Sci. Eng. A 2007, 449-451, 1083-1086. [CrossRef]

39. Chaitoglou, S.; Sanaee, M.R.; Aguiló-Aguayo, N.; Bertran, E. Arc-discharge synthesis of iron encapsulated in carbon nanoparticles for biomedical applications. J. Nanomater. 2014, 2014, 178524. [CrossRef]

(C) 2019 by the authors. Licensee MDPI, Basel, Switzerland. This article is an open access article distributed under the terms and conditions of the Creative Commons Attribution (CC BY) license (http://creativecommons.org/licenses/by/4.0/). 